\title{
Two-Year Follow-up on the Use of Absorbable Mesh Plates in the Treatment of Medial Orbital Wall Fractures
}

\author{
Jae-Pil You, Deok-Woo Kim, Byung-Joon Jeon, Seong-Ho Jeong, Seung-Kyu Han, \\ Eun-Sang Dhong, Woo-Kyung Kim \\ Department of Plastic and Reconstructive Surgery, Korea University College of Medicine, Seoul, Korea
}

Background Absorbable materials offer many advantages in the reconstruction of orbital walls; however, the possibility of postoperative enophthalmos after complete absorption cannot be excluded. We evaluated the postoperative results of absorbable mesh plates used as onlay implanting on the medial orbital wall to determine whether they are suitable for medial orbital wall reconstruction.

Methods The study included 20 patients with medial orbital wall fractures who were followed up for more than 2 years postoperatively. We used absorbable mesh plates in all of the patients. We measured the following: the changes in the expanded orbital volume by comparing the preoperative and postoperative computed tomography (CT) scans and the degree of clinical enophthalmos.

Results There were no major complications associated with the use of absorbable materials such as infection, migration, or extrusion of mesh plates during the long-term follow-up. The orbital volumetric changes between the preoperative and postoperative CT scans were not statistically significant. However, the expanded orbital volume was not related to the degree of clinical enophthalmos.

Conclusions The reconstructed orbital wall may provide supportive scar tissue to the orbital contents even after the absorbable materials have dissolved completely. Absorbable mesh plates could be another option for the reconstruction of the medial orbital wall.

Keywords Absorbable implants / Orbital fractures / Follow-up studies

\author{
Correspondence: Eun-Sang Dhong \\ Department of Plastic and \\ Reconstructive Surgery, Korea \\ University College of Medicine, \\ 148 Gurodong-ro, Guro-gu, Seoul \\ 152-703, Korea \\ Tel: +82-2-2626-1190 \\ Fax: +82-2-852-7484 \\ E-mail: prsdhong@kumc.or.kr
}

This article was at the 70th Congress of Korean Society of Plastic and Reconstructive Surgeons on November 11, 2012 in Seoul, Korea.

No potential conflict of interest relevant to this article was reported.

\section{INTRODUCTION}

There are many different types of materials that can be used in the reconstruction of the orbit. Surgeons can choose either autologous or alloplastic materials, and each material has its own benefits and disadvantages [1]. Absorbable plates and screws have a few advantages over metal plates and screws. These materials undergo biological degradation, and therefore they do not interfere with normal growth and do not need to be removed. However, these materials usually dissolve after 9 to 15 months following insertion and delayed enophthalmos may occur. Also, the use of these materials may trigger a foreign body reaction or 
cyst formation [2].

Al-Sukhun et al. [3] reported that absorbable implants are reliable for large $\left(>2 \mathrm{~cm}^{2}\right)$ inferior orbital wall bony defects. They concluded that absorbable materials provide adequate strength to support the orbital contents during the period of bone healing. However, we found that long-term follow-up computed tomography (CT) showed expanded orbital volume without enophthalmos in some cases.

In this study, we report the two-year follow-up results of onlay implanting of absorbable materials in the treatment of medial orbital wall fractures based on morphometric studies of the orbital wall and morbidity due to enophthalmos. We focused on the following two points: 1) morphometric analysis of the orbit, that is, the volumetric changes in the orbit and the condition of the reconstructed medial orbital wall, and 2) the postoperative clinical enophthalmos.

\section{METHODS}

Between May 2008 and October 2010, 44 patients were diagnosed with isolated medial orbital wall fractures by CT scans. All of the patients had pure medial orbital wall fractures. The patients who had impure orbital wall fractures or combined medial and inferior orbital wall fractures were excluded from the study. Among these 44 patients, 35 were males. The mean age was 31.6 years (range, 15 to 56 years). All of the patients underwent an operation by a single surgeon for reconstruction of the medial orbital wall via retrocaruncular incision with absorbable mesh plates (Inion CPS mesh plate, Inion, Tempere, Finland). Out of the 44 patients, 20 patients were followed up and postoperative CT scans were taken for more than 2 years postoperatively. We calculated the volume of the expanded orbital tissue from CT scans using simple linear measurements [4]. The height of the medial orbital wall defect (a) was measured using the coronal views, the length of the medial orbital wall defect (b) using the axial views, and the degree of medial displacement of the herniated orbital tissue (c) using both the axial and coronal views. The expanded orbital volume through the medial orbital wall defect was measured using this formula (volume $=\pi \mathrm{abc} / 6$ ), assuming that the shape of the dislocated tissue was hemi-ellipsoid (Fig. 1).

We compared the expanded orbital volume between the preoperative CT and postoperative CT. Also, the presence of postoperative enophthalmos was assessed by performing a photographic analysis by two different medical observers. Globe position was assessed grossly by direct visual inspection of the preoperative and postoperative clinical photographs. A worm's-eye view facilitated a gross determination of the axial globe position. The photographs were horizontally compensated before analysis. The evaluation

\section{Fig. 1. Calculation of herniated orbital volume}

$(A, B)$ The herniated orbital volume through the medial orbital wall defect was measured using the formula $\pi a b c / 6$, assuming that the shape of the dislocated tissue was hemi-ellipsoid. a, the height of the medial orbital wall defect; $b$, the length of the medial orbital wall defect; $c$, the degree of medial displacement of the herniated orbital tissue.

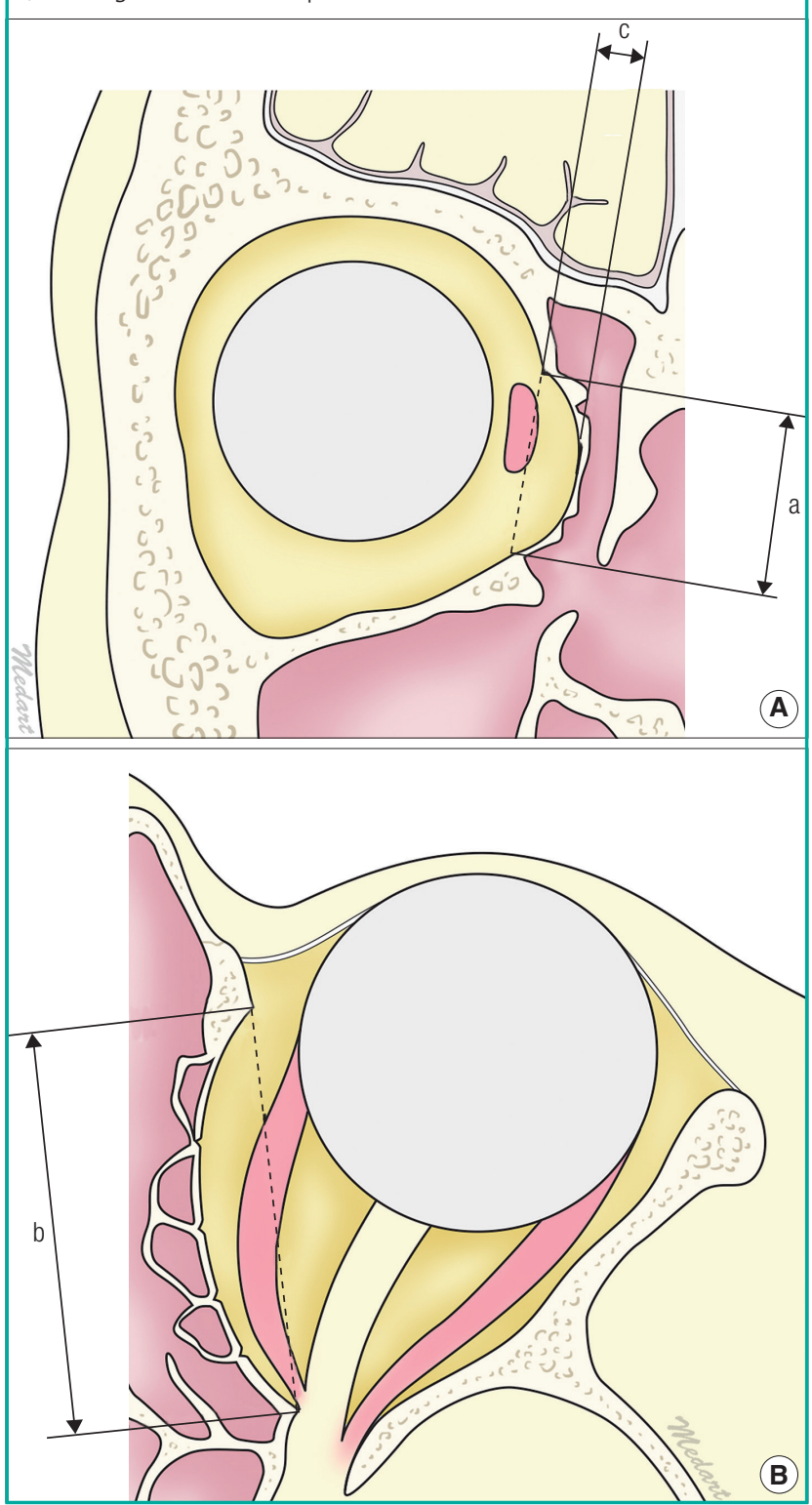

scale consisted of four grades from 0 to 3 , as follows: grade 0 , no enophthalmos; grade 1, mild enophthalmos; grade 2 , moderate enophthalmos; and grade 3 , severe enophthalmos. If two medical observers had different opinions regarding the patient's grade, the average score was used. Hertel's exophthalmometer was used as an ancillary method to measure enophthalmos based on medical records of the ophthalmology department. Analysis of preoperative clinical enophthalmos was not included in this study due to post-traumatic swelling. 


\section{Statistical analysis}

Statistical comparison between the preoperative expanded orbital volume and the postoperative herniated orbital volume was performed using the Mann-Whitney U test. Statistical significance was accepted at the $5 \%$ level.

\section{RESULTS}

None of the patients had complications related to the absorbable mesh plates such as infection, palpable migration, or extrusions of mesh plates during long-term follow-up. We compared the volume of expanded orbital tissue between the preoperative and postoperative CT scans (Table 1). The mean expanded orbital volume was $1.36 \pm 0.88 \mathrm{~mL}$ and $1.32 \pm 0.94 \mathrm{~mL}$ in the preoperative and postoperative CT scans, respectively. Among 20 patients, only one patient (patient No. 2) showed mild enophthalmos postoperatively (Table 2 ). The expanded orbital volume decreased from $1.36 \mathrm{~mL}$ preoperatively to $1.32 \mathrm{~mL}$ postoperatively; however, the difference was not statistically significant $(\mathrm{P}=0.118)$. The postoperative CT scans showed a well healed medial orbital wall, and the integrity of the ethmoid sinus was well preserved. However, there was an overall increase in the orbital volume compared to the normal orbit, and the medial orbital wall was displaced medially (Fig. 2). Postoperative CT scans showed that the medial rectus muscle was located

Table 1. Expanded orbital volume on preoperative and postoperative computed tomography scans

\begin{tabular}{|c|c|c|}
\hline \multirow{2}{*}{ Patient } & \multicolumn{2}{|c|}{ Expanded volume (mL) } \\
\hline & Preoperative & Postoperative \\
\hline 1 & 1.85 & 1.75 \\
\hline 2 & 3.50 & 3.81 \\
\hline 3 & 0.18 & 0.18 \\
\hline 4 & 1.36 & 1.35 \\
\hline 5 & 2.05 & 2.08 \\
\hline 6 & 1.57 & 1.53 \\
\hline 7 & 1.41 & 1.2 \\
\hline 8 & 0.67 & 0.5 \\
\hline 9 & 1.94 & 1.94 \\
\hline 10 & 1.35 & 1.35 \\
\hline 11 & 1.63 & 1.64 \\
\hline 12 & 0.45 & 0.45 \\
\hline 13 & 2.76 & 2.8 \\
\hline 14 & 0.63 & 0.63 \\
\hline 15 & 0.33 & 0.23 \\
\hline 16 & 0.41 & 0.23 \\
\hline 17 & 1.89 & 1.8 \\
\hline 18 & 1.83 & 1.6 \\
\hline 19 & 1.15 & 1.11 \\
\hline 20 & 0.21 & 0.21 \\
\hline Average & $1.362 \pm 0.88$ & $1.324 \pm 0.94$ \\
\hline
\end{tabular}

more symmetrically compared to the preoperative CT scans. The medial rectus muscle was located almost at the homologous position in the reconstructed orbit and the normal orbit. A vague shadow of the scar band was observed between the medial rectus muscle and the reconstructed medial orbital wall (Fig. 2).

\section{Case 1}

A 31-year-old female (patient No. 1) had a left medial orbital wall fracture. The expanded orbital volume was calculated to be approximately $1.85 \mathrm{~mL}$. Nine days after trauma, the swelling improved and the patient showed mild enophthalmos. Medial wall

Table 2. The clinical degree of postoperative enophthalmos was evaluated by medical observers. Hertel's exophthalmometer data was also analyzed

\begin{tabular}{|ccc|}
\hline Patient & Medical observer's grade & Hertel's exophthalmometer $(\mathrm{mm})$ \\
\hline 1 & 0 & $<1$ \\
2 & $1^{\text {a) }}$ & $2^{\text {a) }}$ \\
3 & 0 & 0 \\
4 & 0 & 1 \\
5 & 0 & $1-2$ \\
6 & 0 & 0 \\
7 & 0 & 0 \\
8 & 0 & $<1$ \\
9 & 0 & 0 \\
10 & 0 & 0 \\
11 & 0 & 1 \\
12 & 0 & 1 \\
13 & 0 & 1 \\
14 & 0 & 0 \\
15 & 0 & 1 \\
16 & 0 & 0 \\
17 & 0 & 1 \\
18 & 0 & 0 \\
19 & 0 & $<1$ \\
20 & 0 & 1 \\
\hline a) & 0 & \\
\hline
\end{tabular}

\section{Fig. 2. The location of the medial rectus muscle}

The medial rectus muscle (white arrow) was located at the homologous position in the reconstructed orbit and the normal orbit, and a vague shadow of the scar band (dotted white arrow) was observed between the medial rectus muscle and the reconstructed medial orbital wall.

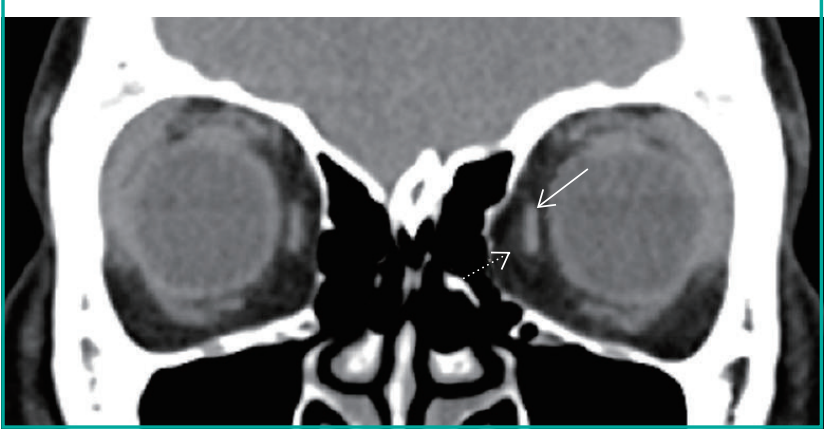




\section{Fig. 3. Case 1, medial orbital wall reconstruction}

(A) Preoperative computed tomography (CT) showed a medial orbital wall fracture on the left side. (B) An immediate postoperative CT scan. The absorbable mesh plate was located in an adequate position. (C) Postoperative CT at the 2-year follow-up showed a well healed medial orbital wall, but both orbits showed asymmetry. (D) Nine days after trauma, swelling subsided and mild enophthalmos was detected. (E) Clinical photograph at the 2-year follow-up showed that enophthalmos was corrected.
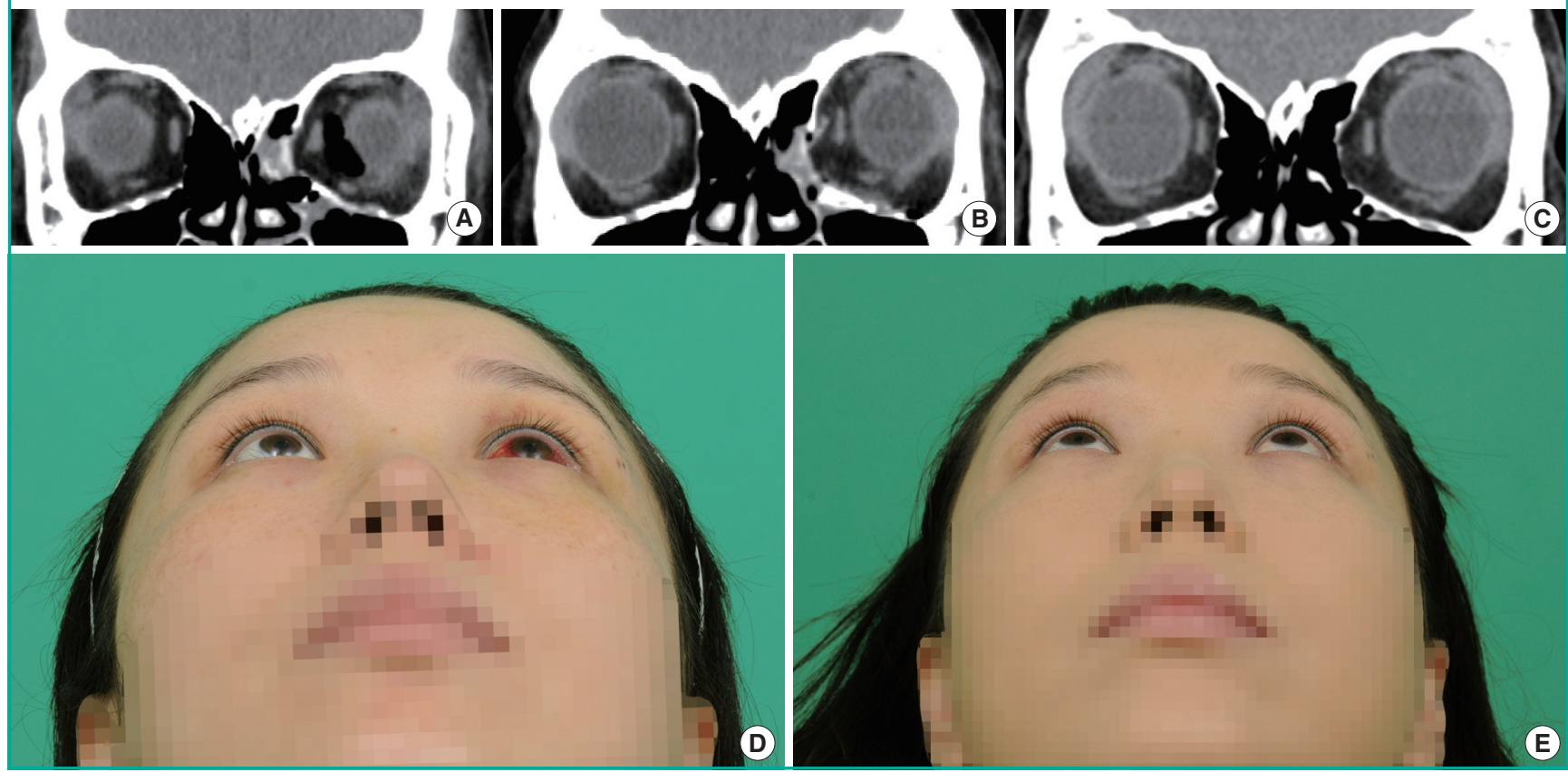

reconstruction with an absorbable mesh plate was performed. The 2-year follow-up CT scan showed an asymmetric orbit but the enophthalmos was corrected (Fig. 3).

\section{Case 2}

A 27-year-old male (patient No. 2) suffered blunt trauma and developed a right medial orbital wall fracture. The expanded orbital volume was approximately $3.5 \mathrm{~mL}$. Eight days after trauma, the medial wall was reconstructed with an absorbable mesh plate. The 2-year follow-up CT scan showed orbital asymmetry and grade 1 enophthalmos was noted at the right side (Fig. 4). Hertel's exophthalmometry showed $2 \mathrm{~mm}$ of mild enophthalmos.

\section{DISCUSSION}

Recently, the incidence of medial orbital wall fracture diagnosis has been increasing due to the invention of diagnostic tools such as CT scans. The importance of surgery for treating medial orbital wall fractures tends to be overlooked because crucial clinical symptoms such as limitation of eye movement are rare. Moreover, it is difficult to assess the degree of enophthalmos because of periorbital edema in the early post-traumatic period. Yab et al. [5] concluded that a relatively good proportional relationship is found between enophthalmos and medial displace- ment of the eyeball, but not between enophthalmos and inferior displacement of the eyeball. Therefore, surgical correction of medial orbital wall fractures is necessary.

Using autogenous materials for reconstruction is a good option; however, donor site morbidity and prolonged operative time are the major concerns [1]. Absorbable materials have many advantages over metal or autologous materials. They are used for pediatric craniofacial surgery because they do not interfere with normal growth. They also do not need to be removed and can be easily contoured when heated. However, the chief concern regarding absorbable materials is infection. Moreover, they usually dissolve after 9 to 15 months of insertion and enophthalmos may occur [2].

Various absorbable materials have been used, and most of the materials are composed of monomers such as $\mathrm{L}$ lactide, $\mathrm{D}$ lactide, glycolide, and tri-methylene carbonate. During the degradation phase, the monomers are metabolized into carbon dioxide and water through the Kreb's cycle [2]. The characteristics and degradation rate depends on the proportion of monomers. Ideal absorbable materials should provide appropriate strength while degrading at an adequate rate without causing any adverse reactions. The overall degradation of Inion does not show the extreme degradation peak that is sometimes seen in products made from one type of polymer (Fig. 5). Therefore, the likelihood of degradation-related inflammatory reactions is usually 


\section{Fig. 4. Case 2, medial orbital wall reconstruction}

(A) Preoperative computed tomography (CT) showed a medial orbital wall fracture on the right side. (B) Postoperative CT at the 2-year follow-up showed a well healed medial orbital wall, but both orbits showed asymmetry. (C) Eight days after trauma, swelling had subsided and mild enophthalmos was detected. (D) Clinical photograph at the 2-year follow-up showed mild enophthalmos of $2 \mathrm{~mm}$, but the patient was satisfied with the results.
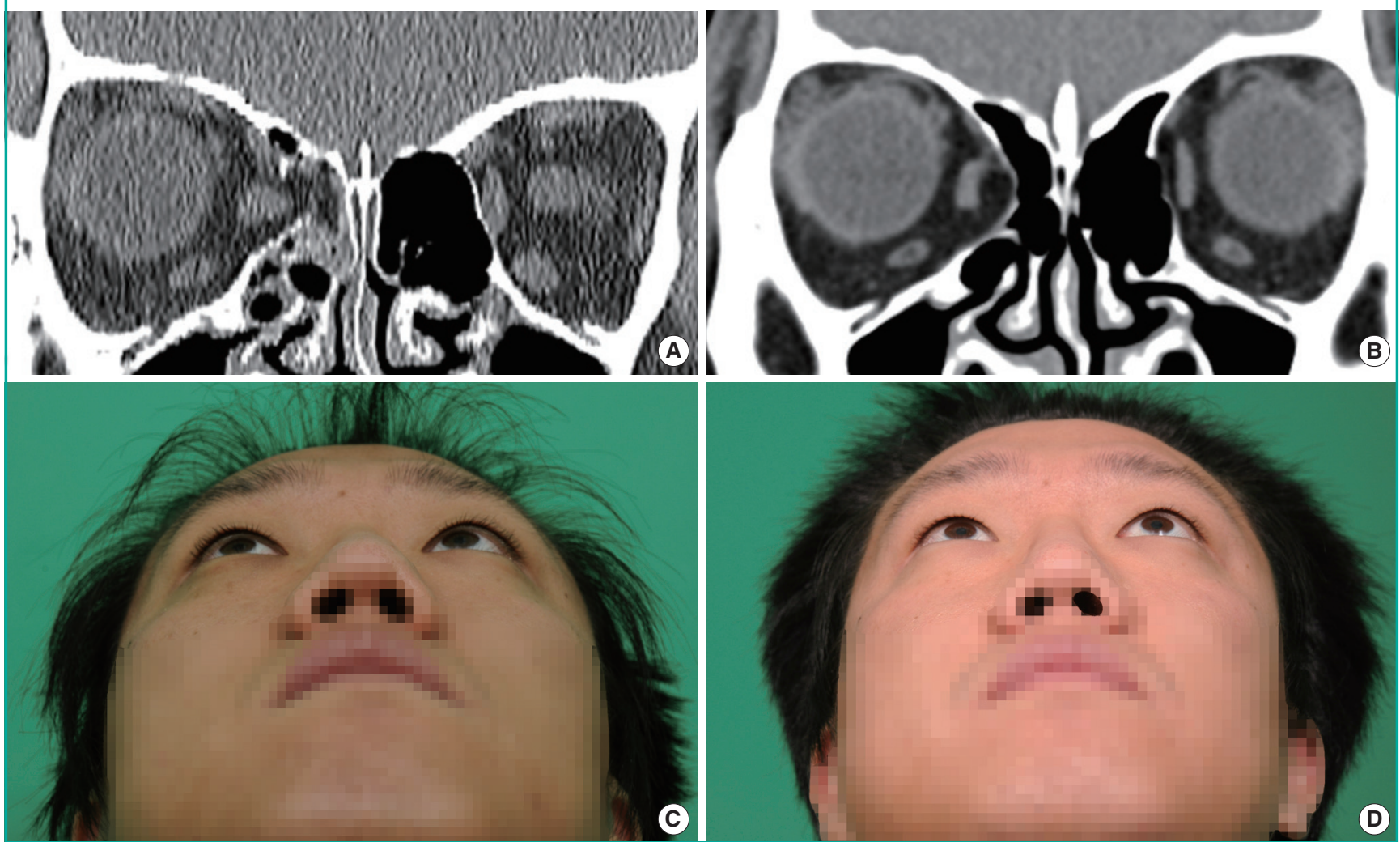

\section{Fig. 5. Degradation rate of absorbable materials}

An Inion mesh plate degrades at an adequate rate compared to fastabsorbing polyglycolide polymer and slow-degrading poly-L-lactide polymer material.

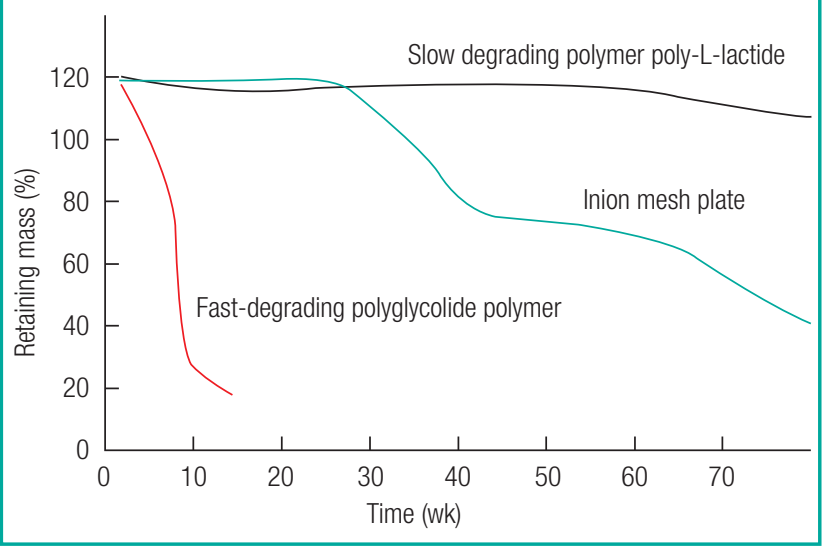

lower than that in the fast-degrading homopolymers, and very low levels of adverse tissue reaction $(-0.1 \%)$ have been reported compared to incidences of up to $46 \%$ seen in other materials [6]. Macrophages and giant cells are responsible for ultimate digestion of the polymeric debris. This is associated with a transient foreign body reaction that is not clinically evident [7]. An adequate degradation rate helps to transfer the load to the healing bone progressively since the material degrades at a rate that coincides with bone healing. In our study, the follow-up CT scans showed well-healed orbital bones, but asymmetric orbits. The reconstructed orbit was expanded compared to the normal orbit on the postoperative CT scan. There was no statistically significant difference between the preoperative herniated orbital volume and the postoperative herniated orbital volume. However, there was one patient who developed enophthalmos during the 2-year follow-up. Al-Sukhun et al. [3] reported that resorption of the absorbable implants seems to take place at a reasonable rate with respect to the bone healing rate in orbital wall fractures. The orbital walls ossified during the follow-up period, and bone healing seemed to take place along the bone fragments. The material showed adequate strength to stabilize the bone segments during the critical period of bone healing, thereby preventing displacement of the overlying orbital contents. Even after the dissolution of the implanted absorbable plate, the medial rectus muscle was located at an almost homologous position in the reconstructed orbit and the normal orbit (Fig. 2). 


\section{Fig. 6. The bony buttress on coronal view}

The bony buttress is identical to the superior medial wall of maxillary sinus. This structure is the bony septum between the maxillary and the ethomoid sinus (arrow). The bony buttress is an important structure to support the medial and inferior orbital walls.

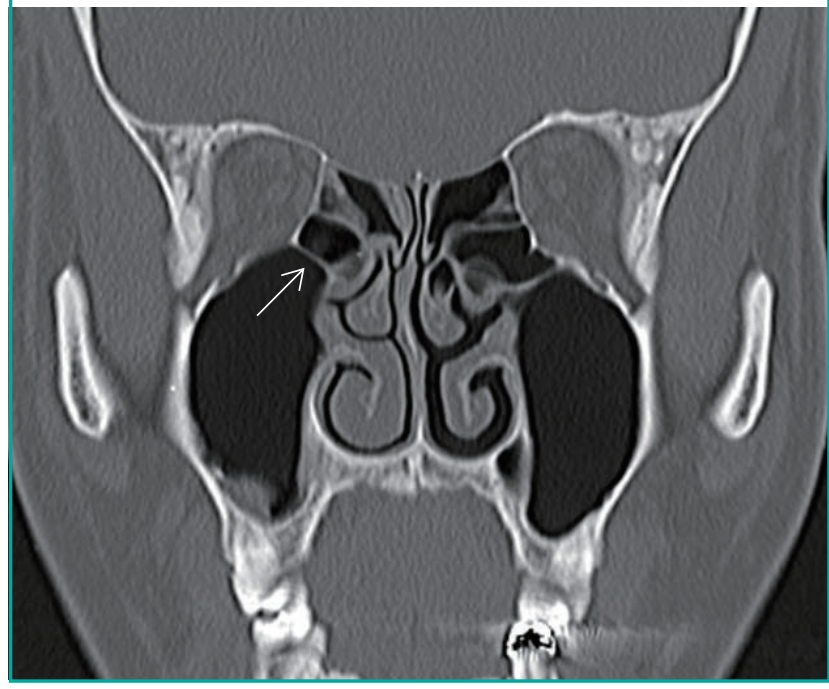

We considered this a sign that the implanted absorbable plate finally provided an adequate supportive scar to the orbital contents like a "hammock" even though the medial bony shadow was displaced medially. On the postoperative follow-up CT scans, increased density was detected like a thin septal pattern at the medial side of the medial rectus muscle. As discussed above, the subclinical inflammatory reaction during biodegradation of the absorbable implants or preserved periosteum may be associated with the formation of a scar band or de novo thin septum.

In our study, an immediate postoperative CT was not conducted in all of the patients. The representative case 1 patient underwent immediate postoperative CT. The immediate postoperative CT showed the absorbable mesh plate was in an adequate anatomical position (Fig. 3). The preoperative expanded orbital volume due to herniation was reduced. However, the long-term follow-up CT showed expanded volume with a fibrous scar band. On long-term follow-up CT, the orbital contents were supported by a fibrous scar band and orbital tissue herniation was not detected. Therefore, absorbable mesh plates might work as internal splints for a fractured orbital wall during their degradation and the bony healing process.

In conclusion, the use of absorbable mesh plates has a number of advantages. The absorbable mesh plate is in an anatomical location immediately after operation. During the absorption of the absorbable material, a fibrous scar band is formed and the medial wall is healed. Even though the volume of the reconstructed orbit is expanded, the reconstructed medial wall prevents orbital tissue herniation and enophthalmos. If the bony buttress fracture (superior medial wall of the maxillary wall) is combined with a medial orbital wall fracture, we prefer to use more rigid materials such as titanium-embedded porous polyethylene or titanium mesh over absorbable mesh plates (Fig. 6). If the bony buttress is collapsed, the supporting system is disrupted and the orbital contents sustain severe injury. Therefore, in such cases more rigid materials are needed [8].

There were some points to consider in our study. First, nowadays, there are many computer software programs that can calculate the expanded orbital volume from CT scans. However, the ethmoid sinus is quite irregular, and unlike the inferior orbital wall, the border of its mucosal surface is not definite. Therefore, to evaluate medial orbital wall fractures, a simple linear measurement is more suitable and can eliminate the subjective bias in measuring the extent of herniation. Second, we mainly used clinical medical observers who graded the cases using photographic analysis to evaluate enophthalmos, and we used Hertel's exophthalmometer as an ancillary method. There are many studies that have used Hertel's exophthalmometer to evaluate enophthalmos. However, Hertel's exophthalmometery is criticized for its low repeatability and low accuracy. Kim and Choi [9] reported that the measurement of exophthalmometric parameters by orbital CT scan could be helpful. However, in our study, we did not use this method. When this method is applied, the axial view is commonly used; however, it is difficult to adjust the midline on the natural head position. Third, further studies with a larger sample of patients are required to establish the effect of absorbable materials with certainty. Further studies using magnetic resonance imaging for follow-up imaging would be very helpful [10].

The authors used the absorbable mesh plate to correct medial orbital wall fracture and obtained fair results such as the following: 1) no major complications, 2) the onlay implanting may provide a supportive scar or de novo septum to the orbital contents even after the absorbable materials have dissolved completely. Absorbable mesh plates could be another option for reconstruction of the medial orbital wall.

\section{REFERENCES}

1. Hollier LH, Rogers N, Berzin E, et al. Resorbable mesh in the treatment of orbital floor fractures. J Craniofac Surg 2001; 12:242-6.

2. Losken HW, van Aalst JA, Mooney MP, et al. Biodegradation of Inion fast-absorbing biodegradable plates and screws. J Craniofac Surg 2008; 19:748-56.

3. Al-Sukhun J, Tornwall J, Lindqvist C, et al. Bioresorbable poly-L/DL-lactide (P[L/DL $] \mathrm{LA} 70 / 30)$ plates are reliable 
for repairing large inferior orbital wall bony defects: a pilot study.J Oral Maxillofac Surg 2006;64:47-55.

4. Jin HR, Shin SO, Choo MJ, et al. Relationship between the extent of fracture and the degree of enophthalmos in isolated blowout fractures of the medial orbital wall. J Oral Maxillofac Surg 2000;58:617-20.

5. Yab K, Tajima S, Ohba S. Displacements of eyeball in orbital blowout fractures. Plast Reconstr Surg 1997;100:1409-17.

6. Bostman O, Pihlajamaki H. Clinical biocompatibility of biodegradable orthopaedic implants for internal fixation: a review. Biomaterials 2000;21:2615-21.

7. Peltoniemi HH, Tulamo RM, Pihlajamaki HK, et al. Consolidation of craniotomy lines after resorbable polylactide and titanium plating: a comparative experimental study in sheep. Plast Reconstr Surg 1998;101:123-33.

8. Burm JS, Chung CH, Oh SJ. Pure orbital blowout fracture: new concepts and importance of medial orbital blowout fracture. Plast Reconstr Surg 1999;103:1839-49.

9. Kim IT, Choi JB. Normal range of exophthalmos values on orbit computerized tomography in Koreans. Ophthalmologica 2001;215:156-62.

10. Cordewener FW, Bos RR, Rozema FR, et al. Poly(L-lactide) implants for repair of human orbital floor defects: clinical and magnetic resonance imaging evaluation of long-term results. J Oral Maxillofac Surg 1996;54:9-13. 